\title{
Modeling Optimization Design of Laser Marking Machine
}

\author{
Xin Hao \\ College of Arts and Design \\ Beijing Forestry University \\ Beijing, China \\ e-mail: 1206998777@qq.com
}

\author{
Jinglian Chen* \\ College of Arts and Design \\ Beijing Forestry University \\ Beijing, China \\ e-mail: jinglianchen@hotmail.com
}

\begin{abstract}
In order to keep up with the growing demand of market, human factors and aesthetics, this paper analyzed and summarized the existing looks of the laser marking machines. Based on the analysis, the author concluded the latest design trends and then verified it by design examples. In this paper, three-dimensional modeling and renderings were created using the modeling software. The study provides a reference for the design of the laser marking machine.
\end{abstract}

Keywords-Industrial design; Optimal design; Laser marking machine; Modeling

\section{INTRODUCTION}

As a modern precision machining method in the electronics industry, laser marking technology is increasingly being accepted by people recently. It has many advantages, like the high quality, high efficiency, non-pollution and low cost, which open up broad prospects for modern processing [1]. LASER is composed of the initials of "Light amplification by stimulated emission of radiation". In 1964, Qian Xue-sen proposed the name "laser", which not only reflects the scientific connotation of "laser stimulated radiation", but also suggested that it is a very strong new light.

With the growing use of modern laser marking technology, the demand for miniaturization, high efficiency, integration and aesthetic becomes more heavy. On the contrary, most of the domestic laser marking machines originally are monotonous in modelling, rough in manufacturing processes and old in color. Instead of high-tech, high-quality and sophisticated instruments, they look cold, heavy and crude, which is certainly incompatible with the rapid development of laser marking technology.

By analyzing the characters and disadvantages of the original design of laser marking machines, the authors explored the relationship between the requirements and the looks, and then designed a new laser marking machine based on the research. The original laser marking machine is shown in "Fig. 1".

\footnotetext{
* Corresponding author: Jinglian Chen.

The current study was supported by the Fundamental Research Funds for the Central Universities (YX2013-25).
}

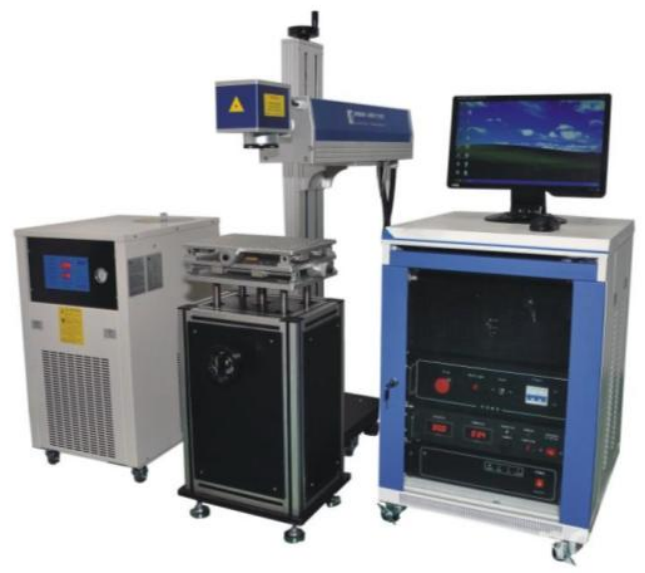

Fig. 1. The original laser marking machine.

\section{INTER PRODUCT STATUS ANALYSIS}

\section{A. Operational Principle}

Laser marking machines uses high energy density lasers to print sharp and clear graphics, text, bar codes and other information on the surfaces of the paper, metal, plastic, glass, wood and so on. Compared with electrical discharge machining, mechanical characterization, printing and other traditional processing methods, laser marking technology has unparalleled advantages. For example, laser controls space and time well and it is capable to deal with projects with most kinds of materials, shapes and sizes in different processing environment while using materials more efficiently; laser portrays better lines, which can reach millimeters to microns; laser processing is a clean and environmental friendly processing technology without pollution. Therefore, laser printing technology has been widely used in various industries

With the growing use of modern laser marking technology, the demand for miniaturization, high efficiency, integration and aesthetic becomes more and more heavy.

Most laser marking machines are split into several independent parts. They are composed of a laser head, main control cabinet and work rack. Among them, the main control cabinet is large, and must be operated with a control panel, a 
keyboard and a monitor. So the operation is complex. Light gun is composed of a laser head and laser generator; main control cabinet includes control cabinet, keyboard, control panel and monitor; work rack includes rack shelves and casters. Its structure is shown in "Fig 2".

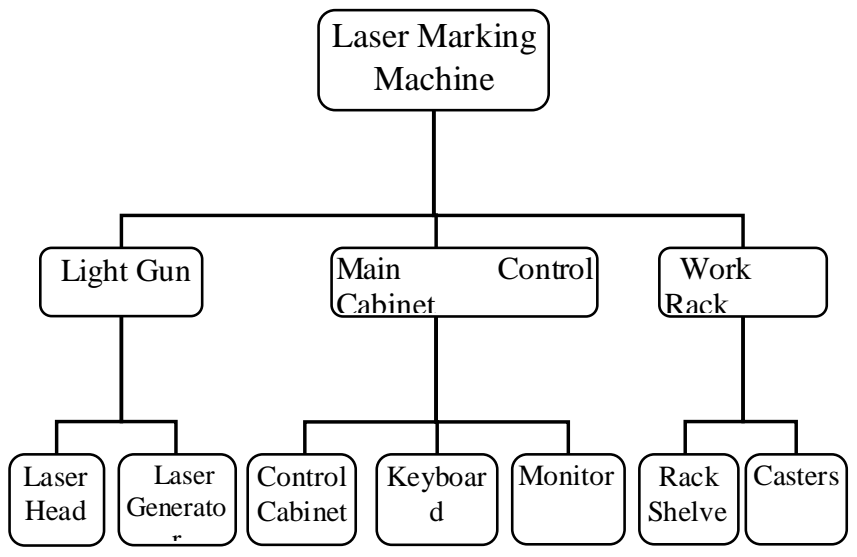

Fig. 2. Structure of the original laser marking machine

\section{B. The Original Design Flaws}

Laser marking machines originally are split, dull and having poor appearances. But as a kind of product with a high technical content, it should look high-tech and sophisticated. Besides, the original laser marking machine design usually doesn't take into account aesthetic and ergonomics. Design flaws analysis is shown in "Table 1".

TABLE I. ORIGINAL LASER MARKING MACHINE DESIGN FLAWS

\begin{tabular}{|c|l|}
\hline Design elements & \multicolumn{1}{c|}{ Design Flaws } \\
\hline Shape & $\begin{array}{l}\text { old-fashioned, abruptly transitional, lacking modeling } \\
\text { semantic, lacking in the sense of high quality and } \\
\text { sophistication }\end{array}$ \\
\hline $\begin{array}{c}\text { Man-machine } \\
\text { relationship }\end{array}$ & $\begin{array}{l}\text { large and not portable, lacking operating instructions } \\
\text { and safety, unergonomic }\end{array}$ \\
\hline Color & dull, lacking clear indicative color and logo \\
\hline
\end{tabular}

\section{PRODUCT OPTIMIZATION DESIGN IDEAS}

The design of laser marking machines on the market now are monotonous and not integrated. And the costs of Installation, packaging and transportation can be high. So the appearance design optimization of laser marking machines has an important significance. With a good layout, the product can maximize the effect of its functions [2] 错误! 未找到引用 源。. In addition, the original laser marking machine is difficult to disassemble and clean, and the appearance is not good enough either. Therefore, when optimizing the laser marking machine modeling, three aspects should be emphasized: shape, man-machine relationship and color.

\section{A. Shape Optimization}

When operating laser marking machines in the marking workshop, engineers and workers have to face non-stop assembly lines and process the same kind of product repeatedly for a long time. Inevitably, this work will make people feel dull and boring. Besides, the design of laser marking machines in the market nowadays has few differences. The monotonous and abrupt shapes, the cluttered interface and the dull work processes are easy to cause operators fatigued, let alone comfort and humane of work. If a laser marking machine which has a better shape and a more simple interface is created, operators will obtain a new pleasant experience when working. This will certainly increase the market competitiveness of the product.

\section{B. Ergonomics Optimization}

The market of laser marking machines is increasingly competitive as the technology matures. However, the laser marking machines currently still face a series of problems. Their functions are simple and their interfaces are not friendly. For example, most of the domestic laser marking machines dominated by stationary powerful marking, one type of modeling is not easy to move, and it is difficult to achieve dynamic marking. However, with the development of laser technology, the integration of control source and laser generator has been achieved. And this makes miniaturized and portable design of laser marking machines become possible. Besides the above, the operating instructions and safety of laser marking machines also need to be improved.

\section{Color Optimization}

The color of the current laser marking machines is dull. There is no clear color system and the environmental integration is poor. Moreover, the product cannot reflect the characteristics of the laser marking machine industry. The product optimization design should follow the aesthetics laws to match colors. The product color should suit the environment and look modern. And it has to be indicative and easily identifiable to strengthen security.

\section{LASER MARKING MACHINE OPTIMIZATION DESIGN}

\section{A. Good Function Experience}

A good function experience requires designers to dig deeper and go beyond the obvious demand of users. The study should focus on usage scenario to deeply understand the expectations of operators. The optimized laser marking machine is versatile. It has the function of both fixed marking and dynamic marking. During the design, the designer always focused on the function element "Portability", combining control source, control cabinets and laser generator into a coherent whole through integrated design. This minimized the volume and made it easy to store and move. In addition, the control terminal of the laser marking machine changed from the big screen control cabinet into a handheld touch screen controller, and operation changed from the traditional keyboard and mouse input. 


\section{B. Rich Modeling Language}

Besides the fulfilment of versatility, the shape of new laser marking machine also broke through the conventional styling. It replaced founder, old-fashioned features with smooth organic shapes full of energy and affinity. The overall shape consisted of two blocks of contrasting colors. And the blocks weaved together with soft arc transitions, avoiding making users feel mechanical, cold and heavy. The new shape was full of changes and rhyme. The contrasting color of black and white goes with the characteristic features of the product, and symmetric design is used here to make the product more perfect. The dark block in the middle highlights the functional area and its prominent shape makes the product looks much lighter and slimmer. The stage of product appearance optimization requires computer-aided design, and Rhino and Solidworks 错误! 未找到引用源。 were used to create a three-dimensional modeling with all the details. The final plan is an integrated modeling. The 3D modeling is shown in "Fig. 3 " and the rendering is shown in "Fig. 4".

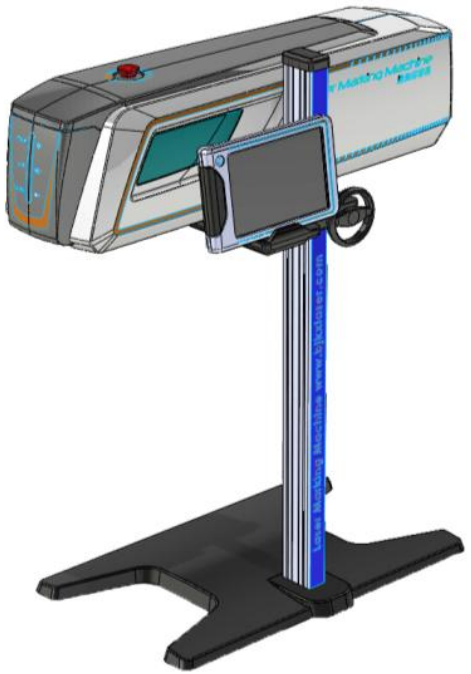

Fig. 3. Integrated laser marking machine modeling

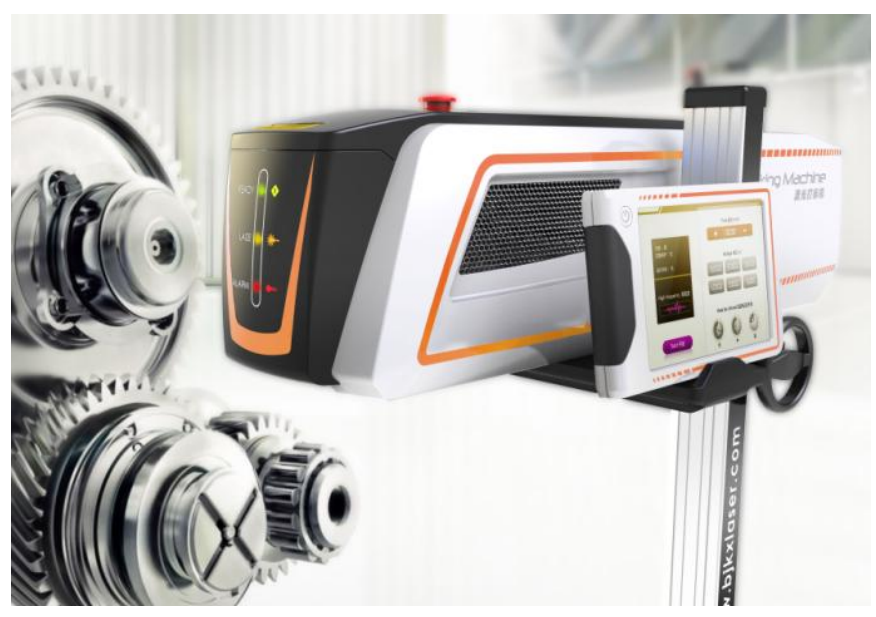

Fig. 4. Integrated laser marking machine rendering

\section{Emotional Experience}

Products exist for users. Hence we need to analyze and study the aesthetic taste of users, and add a feeling of affinity to user experience. A good product impressed people by making the product interact with users emotionally [5]. The appearance design of the new laser marking machine is based on modern railway, which is familiar to people. The design combines the physical form factors of railway with laser marking machines, revealing the character of quickness, accuracy and efficiency, fully showing the products and brands value. And the design resonates with operators well. The orange decorative trim creates a vibrant look for the product, bright but not jarring, acting as a useful warning to some extent. The decorative trim was " $\mathrm{v}$ " shaped, meaning "victory", reflecting the high quality, fast and accurate characteristics of the laser marking machine industry. This fully conveys the emotion and energy of the design. The decorative trim shown in "Fig. 5".

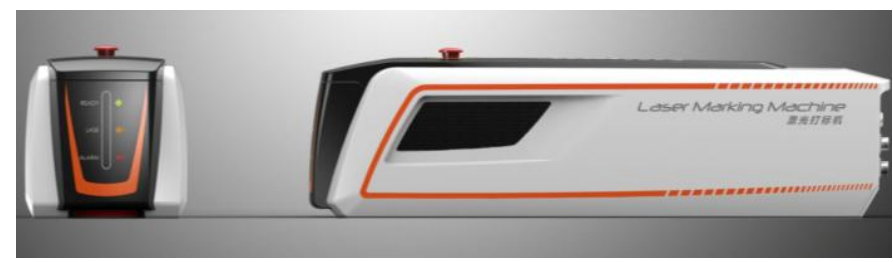

Fig. 5. Laser marking machine decorative trim

\section{Design Details}

The laser generator is integrated and has a work rack with wheels in order to move easily and achieve dynamic marking. On the work rack, there is a shelving rack used to store and manage the controller. The detail design of the shelving rack is shown as the "Fig. 6 (a)".

The large-screen controller interface is more convenient. It has more functions and better performance. The Indicator is designed to visually show the working condition of the machine to ensure safe operation. The detail design of the indicator is shown as the "Fig. 6 (b)".

The emergency stop switch has adopted a chain operation pattern of press - rotation in order to effectively prevent accidental collisions caused by misuse. This change can reduce losses due to machine error stop and pipeline resume debugging. The detail design of the emergency stop is shown as the "Fig. 6 (c)".

\section{CONCLUSIONS}

The design focuses on appearance optimization and function integration of laser marking machines. Through the design, the appearance and the operation of the product have been improved in shape, man-machine relationship and color. The theory of industrial design is used to enhance the added value for the products. It saves costs of manufacturing, equipment, packaging and transportation, and improves the aesthetic, innovation and the man-machine operation for the laser marking machine. This optimization design method applies not only to laser marking machines, but also can provide a reference for optimization design of related products. 


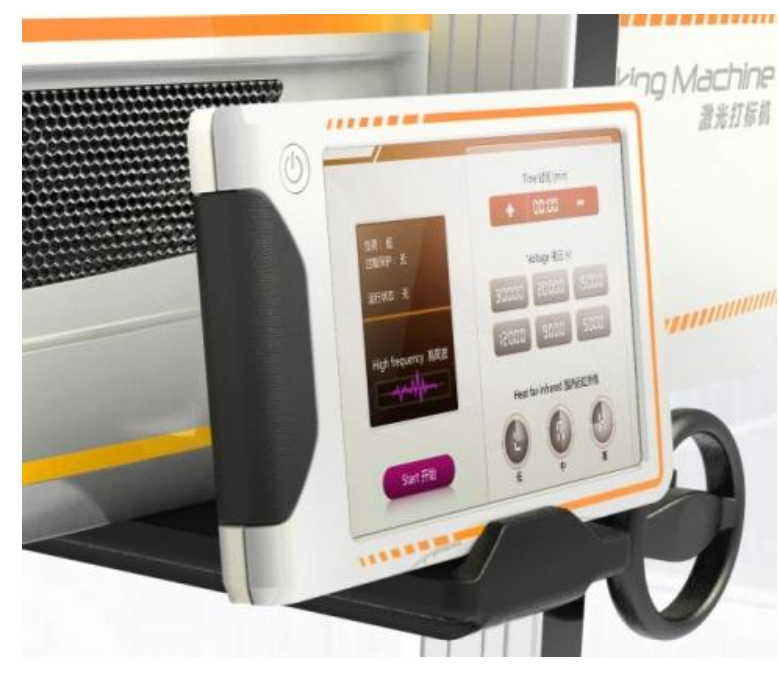

(a)



(b)

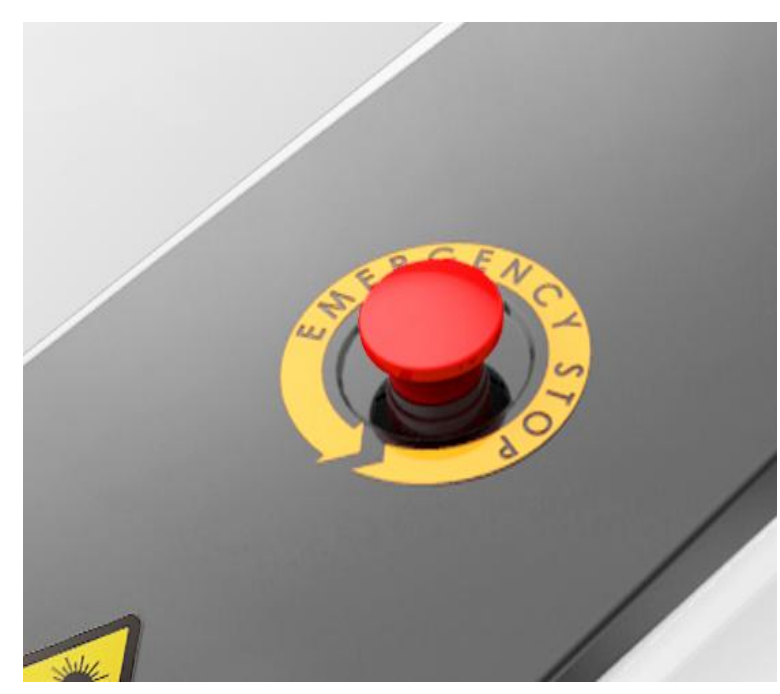

(c)

Fig. 6. Details of the laser marking machine.

\section{ACKNOWLEDGMENT}

The current study was supported by the Fundamental Research Funds for the Central Universities (YX2013-25).

\section{REFERENCES}

[1] Zhang Yi. Marker innovative design based on Kansei Engineering Research playing laser $[\mathrm{J}]$, Huazhong University of Science and Technology master's degree thesis, 2011 (1): 2-3.

[2] Gerda Gemser, Mark A A M. How integrating industrial design in the product development process impacts on companyperformance[J].The Journal of Product Innovation Management, 2001(18): 28-38.

[3] Fu Zhi-guo, Luan Li-juan, Zhang Yan-ping. Quality-oriented engineering product design fuzzy evaluation [J]. Mechanical design, 2014, 3 (2): 111-114.

[4] Zhang Yin. Application of three-dimensional modeling of the midpoint of the Rhinoceros [J]. Mechanical design, 2013, 30(10): 106-110.

[5] Li Nan. Product Innovation Based on user experience redesign [J]. Furniture and interior decoration, 2010 (4): 90-91. 